\title{
The phase diagram of a gauge theory with fermionic baryons
}

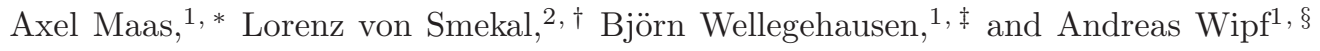 \\ ${ }^{1}$ Theoretical-Physical Institute, Friedrich-Schiller-University Jena, Max-Wien-Platz 1, D-07ry3 Jena, Germany \\ ${ }^{2}$ Institut für Kernphysik, Technische Universität Darmstadt, D-64289 Darmstadt, Germany
}

(Dated: April 1, 2019)

\begin{abstract}
The fermion-sign problem at finite density is a persisting challenge for Monte-Carlo simulations. Theories that do not have a sign problem can provide valuable guidance and insight for physically more relevant ones that do. Replacing the gauge group SU(3) of QCD by the exceptional group $\mathrm{G}_{2}$, for example, leads to such a theory. It has mesons as well as bosonic and fermionic baryons, and shares many features with QCD. This makes the $\mathrm{G}_{2}$ gauge theory ideally suited to study general properties of dense, strongly-interacting matter, including baryonic and nuclear Fermi pressure effects. Here we present the first-ever results from lattice simulations of $\mathrm{G}_{2} \mathrm{QCD}$ with dynamical fermions, providing a first explorative look at the phase diagram of this QCD-like theory at finite temperature and baryon chemical potential.
\end{abstract}

PACS numbers: 11.30.Rd 12.38.Aw 12.38.Gc 12.38.Mh 21.65.Qr

Finite fermion density continues to be a serious challenge for Monte-Carlo simulations due to the fermionsign problem [1, 2]. The sign problem appears in many areas of physics, but is of notorious importance to dense quark systems, especially in nuclei, heavy-ion collisions, and compact stellar objects. An alternative are models and continuum methods which do not have this type of problem [3 7]. However, these usually require approximations, and cross checks through lattice simulations remain desirable to improve systematic reliability.

To provide support from numerical simulations, two major strategies have been followed. One is to replace the baryon chemical potential by some quantity more amenable to simulations, e.g. imaginary [8 10] or isospin [11, 12] chemical potential. The other is to replace the theory with one accessible through numerical simulations at finite density. However, such theories usually differ from the original one in more or less important aspects.

One very well studied replacement of QCD for strongly interacting matter at finite density is two-color QCD $13-$ 17]. In this case, the baryons are bosons instead of fermions, however. This leads to profound differences, such as Bose-Einstein condensation of a baryon superfluid with a BEC-BCS crossover at high densities instead of the usual liquid-gas transition of nuclear matter. While two-color QCD has many interesting aspects that deserve to be studied in their own right, the quantum effects due to the fermionic nature of baryons are expected to play a very significant role for nuclear matter and especially in the physics of compact stellar objects [18].

Therefore, a more realistic replacement theory in this regard should contain fermionic baryons. One possibility is the strong-coupling limit [19]. In order to maintain

\footnotetext{
*Electronic address: axelmaas@web.de

${ }^{\dagger}$ Electronic address: lorenz.smekal@physik.tu-darmstadt.de

¥Electronic address: bjoern.wellegehausen@uni-jena.de

$\S$ Electronic address: wipf@tpi.uni-jena.de
}

the connection with the continuum, however, we employ here a different theory without sign problem for MonteCarlo simulations. It is obtained by replacing the $\mathrm{SU}(3)$ gauge group of $\mathrm{QCD}$ with the gauge group $\mathrm{G}_{2}[20]$. All color representations of this theory are equivalent to real ones. As a consequence the Dirac operator has an antiunitary symplectic symmetry which for $N_{f}$ flavors leads to an extended Pauli-Gürsey $\mathrm{SU}\left(2 N_{f}\right) \times \mathrm{Z}_{2}$ flavor symmetry [13, 20], and is thus expected to have a non-anomalous component even for a single flavor. In this paper we study the phase diagram for a single Dirac flavor of Wilson fermions, corresponding to a continuum $\mathrm{SU}(2) \times \mathrm{Z}_{2}$ extended flavor symmetry in the chiral limit. Spontaneous or explicit breaking reduces this to $\mathrm{U}(1) \times \mathrm{Z}_{2}$ [21]. The unbroken $\mathrm{U}(1)$ relates to the baryon number to which the baryon chemical potential is coupled. The anti-unitary symplectic symmetry of the Dirac operator in a real color representation implies a two-fold degeneracy of its eigenvalues and hence that the fermion determinant remains positive at finite baryon chemical potential even for a single flavor [13, 14].

The physical bound states of this theory, besides the usual quark-antiquark and three-quark states, also contain hybrids of one quark with three gluons, as well as diquarks and further bound states with more than three quarks [20]. Thus, the hadronic spectrum contains both fermionic as well as bosonic baryons and mesons. These bound states are created by very similar interactions as in $\mathrm{QCD}$, i. e. by a potential which rises linearly with the separation of the quarks before string-breaking sets in [20, 22 25]. In fact, in a simple quark model picture with light quarks, one has a baryon-mass hierarchy where for a sufficiently small quark mass nucleonic three-quark states will be the second lightest baryons, above the light diquarks as the would-be-Goldstone bosons of the extended flavor-symmetry breaking. Hybrids, tetraquarks and other baryonic multi-quark states are expected to be much heavier.

Another appealing aspect of the $\mathrm{G}_{2}$ gauge group is that it might be deformable to ordinary QCD by break- 

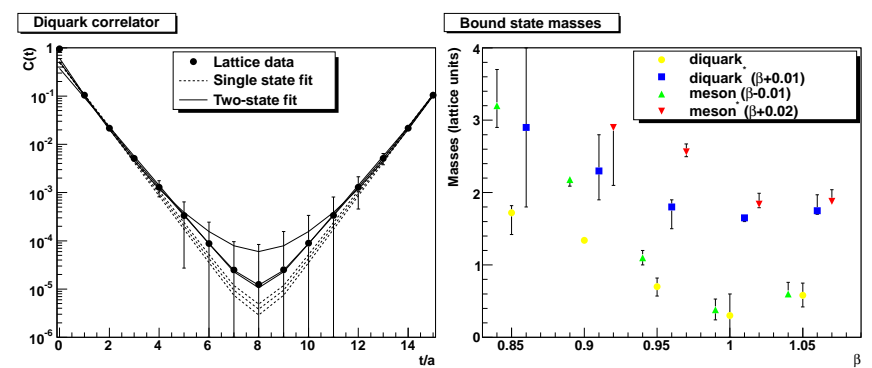

FIG. 1: The left panel shows an example for the mass correlator fits performed. Since in most cases no clear mass plateaus appear due to closeness of the excited states, conservative error estimates have been used, which are shown in the example as error bands. The right panel shows the masses obtained in this way. Note the shifted $\beta$ values to disentangle the plot. All results are for $\kappa=0.15625$. Only the range $\beta=0.9$ to $\beta=1.0$ has been used in the phase diagram calculations.

ing $\mathrm{G}_{2}$ down to $\mathrm{SU}(3)$ via a Higgs mechanism [20, 26], although this will likely require several Higgs fields and various Yukawa couplings, CKM-type explicit flavor violations, and further effects. The surplus bound states would then become heavy and disappear from the spectrum. The sign problem would emerge, likewise. If it can be controlled by the strength of the breaking, however, this could provide new insights, a possibility that certainly deserves further study in the future.

For our simulations, which consumed roughly 2000 core-years, we employ an extension of the available local HMC algorithm for scalars 26], generalized from QCD simulations, for details see [21, 27]. The introduction of temperature and chemical potential proceeds as in QCD. Our aim here is a first exploration of the phase diagram as a proof of principle. One obstacle is the presence of a bulk transition [24, 26, 28], which turns out to persist with dynamical fermions [21, 27]. To avoid this we chose a lattice with at least $N_{t}=5$ time slices in finite temperature simulations. Simulations of this theory incur considerable computational costs so that we restricted our lattices to $N_{s}=16$ points in spatial directions. Altogether, we investigated three different sets of lattice parameters:

(a) At zero density we varied the lattice coupling $\beta$ between 0.9 and 1.0 in order to control temperature on our $5 \times 16^{3}$ and $6 \times 16^{3}$ lattices.

(b) At finite chemical potential $\mu$ we used $\beta=0.9$ on both, $6 \times 16^{3}$ and $8 \times 16^{3}$ lattices, and $\beta=1.0$ in zerotemperature simulations on a $16^{4}$ lattice. The hopping parameter in the fermion determinant was fixed at $\kappa=$ 0.15625 in all these thermodynamic simulations.

(c) For comparison we also varied $\kappa$ from 0.15385 to 0.15625 on the symmetric $16^{4}$ lattice with $\mu=0$ and $\beta$ in 0.85 to 1.1. [21].

More results from smaller lattices to assess systematic effects will be reported elsewhere.

We measured three observables to study the phase diagram. One is the Polyakov loop. Unlike QCD, due to the trivial center, it is not an order parameter of the

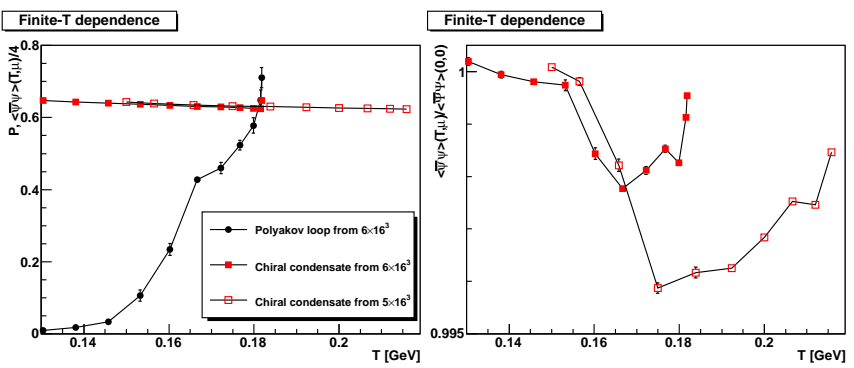

FIG. 2: The Polyakov loop and the unrenormalized chiral condensate at zero chemical potential (left panel) and the renormalized chiral condensate (right panel).

quenched theory [20], but it nevertheless reflects the corresponding first-order phase transition very well [24, 28]. In fact, it remains so small in the low-temperature phase that it is only possible to determine upper bounds. We found this to be true also with dynamical quarks. The second observable is the chiral condensate. The quenched $\mathrm{G}_{2}$ theory has only one first-order transition at finite temperature which manifests itself also in the chiral condensate [29], a feature that it shares with QCD and two-color QCD. This is in contrast to QCD with adjoint quarks, where there is no sign problem either 13], but where separate chiral and deconfinement transitions occur at largely different temperatures 30 32]. We normalize the chiral condensate to its ( $\beta$-dependent) vacuum value, to avoid explicit renormalization. The third observable is the baryon number density, the derivative of the partition function with respect to the chemical potential. At large chemical potentials the density saturates to a temperature-independent value. This is observed also in two-color QCD [33]. To eliminate the scale, we therefore normalize the baryon density to its saturation value.

Saturation on finite lattices can occur due to the Pauli principle. In two-color QCD, this happens when all sites are occupied by bosonic baryons such that the lattice has the maximal filling of $4 N_{f}$ quarks per site as allowed by the Pauli principle [15]. Here, we also find the analogous saturation at a maximum of 14 quarks per lattice site for the two spin states of a single quark flavor in the 7 dimenisonal fundamental color representation of the $G_{2}$ gauge group. However, the fermion dynamics does not fully freeze out because the plaquette average at saturation density is $0.5647(1)$, which is smaller than the corresponding value of $0.5724(5)$ for the pure gauge theory.

In order to assess the phase diagram it is necessary to fix at least a relative scale. Using bound state masses for this purpose is rather challenging. We have determined the masses of the lowest states in the scalar meson and diquark channels on the $16^{4}$ lattice, see Figure 1. These include only the connected contributions, however. The true scalar meson in our one-flavor case contains disconnected contributions as well which will cause the dominant splitting of its mass from that of the light diquark. The results show strong systematic effects 21]. Moreover, the diquarks are most sensitive to the quark mass 


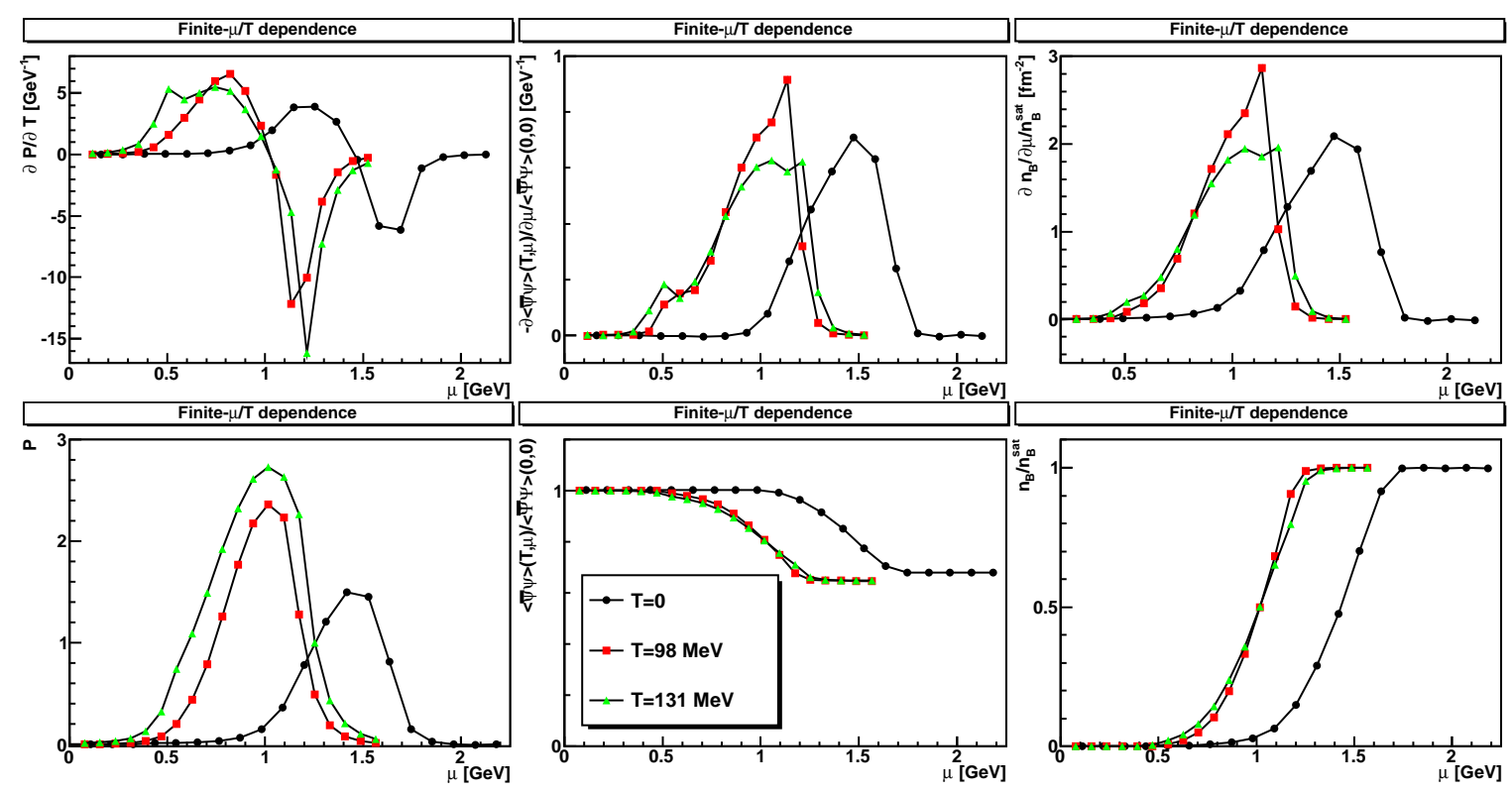

FIG. 3: Bottom row: raw Polyakov loop data (left), chiral condensate (middle), and normalized baryon density (right) at finite quark chemical potential $\mu$ and temperatures $T=0,98 \mathrm{MeV}$ and $131 \mathrm{MeV}$. Top row: corresponding numerical derivatives.

due to their would-be-Goldstone nature [34]. In order to fix the scale from these masses would require a much more careful and expensive mapping of the lines of constant physics in $\beta$ and $\kappa$. Here we simply use the first excited state in the diquark channel as the signal with the least sensitivity to the lattice parameters. It varies for $\beta=0.9 \ldots 1.0$ at $\kappa=0.15625$ from $2.3_{-4}^{+5}$ to $1.65_{-5}^{+1}$ in lattice units. Requiring that the critical temperature for $\mu=0$ is at $160 \mathrm{MeV}$ then leads to lattice spacings between $0.25_{-4}^{+5} \mathrm{fm}$ to $0.181_{-20}^{+1} \mathrm{fm}$ in the same range for $\beta$, with an acceptable systematic uncertainty for this first exploratory investigation.

The results at zero chemical potential are shown in Figure 2. The normalized chiral condensate only shows a rather weak response to the transition observed in the Polyakov loop. In fact, after a slight drop the chiral condensate starts rising again as temperature is further increased. That this behavior is likely to be a lattice artifact is seen in particular by comparing the data from the $5 \times 16^{3}$ and $6 \times 16^{3}$ lattices as shown for the same range of couplings $\beta=0.9 \ldots 1.0$ at $\kappa=0.15625$ in Fig. 2.

On one hand, this apparently unphysical behavior of the condensate might be due to finite volume effects, because the three-dimensional volume shrinks with increasing temperature when controlled by the lattice coupling as it is done here. On the other hand, in the zero temperature simulations we observe that the meson and diquark masses at fixed $\kappa=0.15625$ start increasing with $\beta$ again just above $\beta \approx 1$, c.f. Fig. 11 This might indicate that we encounter a transition into an unphysical phase such as the Aoki phase 21]. Although we have not carefully mapped out the lines of constant physics, for the highest temperatures in Fig. 2. which correspond to $\beta=1$, we might be getting too close to or even into this unphysical phase. Both, finite-volume effects and Aoki phase would lead to an unphysical behavior as observed, a weakening of the chiral transition together with an increase of the chiral condensate towards higher temperatures.

While this implies that even larger and finer lattices will be required to reliably quantify the systematic uncertainties in the high temperature regime, we avoid these lattice artifacts in our finite density simulations below as much as possible with controlling temperature by varying the number of time slices at reasonably small $\beta$ values. From our present analysis we are confident that the lattice parameters (b) used at finite chemical potential, as described above, should be reasonably safe. A more detailed quantitative analysis, in particular at temperatures near the $\mu=0$ transition in Fig. 2 and above, seems prohibitively expensive at the moment.

As a final comment on the $\mu=0$ transition before we discuss our finite density results we note that, strictly speaking, there is no chiral symmetry in the usual sense in our one-flavor theory. Rather, the condensate here measures the breaking of the extended SU(2) Pauli-Gürsey symmetry down to the usual baryon number U(1). Thus, our intuition about the usual chiral restoration at the deconfinement transition might not be a good guide in this case either. It is quite possible, for example, that the 2 -flavor theory behaves differently because it includes a standard chiral $\mathrm{SU}(2) \times \mathrm{SU}(2)$ component in its extended flavor SU(4) which is expected to be broken at low temperatures and which might therefore show a stronger response to the $\mu=0$ transition in $G_{2}$ as well.

The behavior of Polyakov loop, chiral condensate and quark density at finite chemical potential with the scale set as explained above and in lattice units are shown in Figures 3 and 4 At zero temperature the Polyakov loop peaks at around $1.5 \mathrm{GeV}$. Chiral condensate and baryon density both show a rather broad transition near 

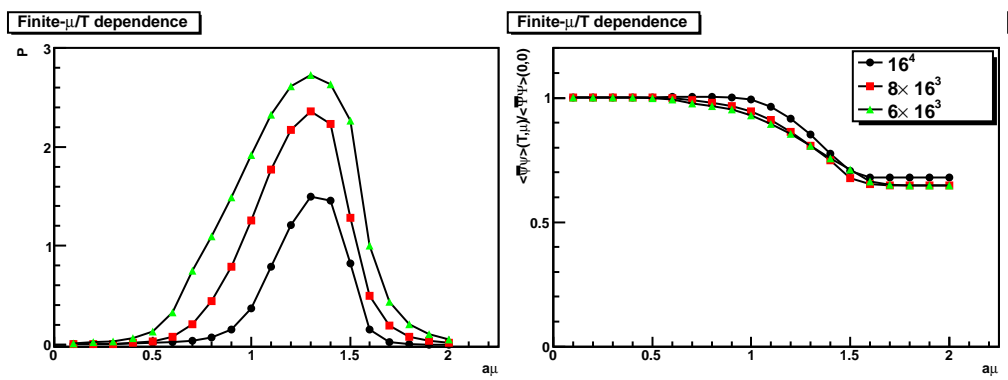

Finite- $\mu / \mathrm{T}$ dependence

FIG. 4: The data from the bottom row of Figure 3 in lattice units.

the maximum in the derivative of Polyakov loop. At large chemical potential the density saturates, as discussed above. This effectively quenches the theory which explains the decrease of the Polyakov loop as in two-color QCD [15, 33]. It also implies, however, that the regime beyond the peak in the Polyakov loop is affected by lattice artifacts thus limiting the chemical potential to below $1.5 \mathrm{GeV}$ at $T=0$ and to below about $1 \mathrm{GeV}$ at the two finite temperatures considered here. This is emphasized by the result in lattice units where the saturation is seen to be nearly independent of the lattice coupling.

In the zero temperature results for the quark density one should see the onset of Bose-Einstein condensation of diquarks when the quark chemical potential reaches half the diquark mass. With a mass of the lightest diquark around $300-400 \mathrm{MeV}$ here, this so-called silverblaze point [35] is thus expected around $\mu \approx 150-200$ $\mathrm{MeV}$. On smaller $16 \times 8^{3}$ lattices, with larger masses and with considarably better statistics, one indeed observes this onset of diquark condensation in the baryon density which increases from zero to a small finite plateau in a narrow region [21]. The critical chemical potentials for this onset furthermore agree very well with half the diquark masses as extracted from their correlators for a wide range of lattice parameters 21, 36]. It is a small effect, however. So small that it cannot be seen on the scales of the Figures presented here. A more detailed study with finer resolution on the present lattices requires much higher statistics. Having said that, the onset of the large increase in density observed here, for $T=0$ at $\mu \approx 1 \mathrm{GeV}$, is by the same argument unlikely to be due to these light pseudo-Goldstone diquarks. The first excited diquark states of Fig. 1 1 should come in at $\mu$ around $400-550 \mathrm{MeV}$ and thus still tend to be too small to explain this threshold. The intriguing alternative would be that we do see the effect of fermionic baryons here. If the quark-gluon hybrids and other baryons are too heavy as expected, one would conclude that the density in the region around the increase at $\mu \approx 1 \mathrm{GeV}$ is dominated by three-quark states, the $G_{2}$ nucleons. We will find out with further baryon spectroscopy in the future.

Finally, when both temperature and chemical potential are non-zero, a gradual shift towards smaller chemical potentials along with a broadening of the transition is observed. The interpolated phase diagram in Figure 5 exhibits a rather polygonal shape but is potentially af- fected by systematic effects in the scale setting. Results on smaller lattices show the same general features albeit with larger systematic errors [21].

In total, the phase diagram of $G_{2}$ QCD shows a number of interesting features which deserve further systematic investigation to see whether they are genuinely physical, or whether some of them are lattice artifacts. Given sufficient computational resources its complete phase diagram can be mapped with any desired precision. In particular, the question whether one can have a chiral first-order transition rather than a smooth crossover at finite density is very significant as it would imply the presence of a critical endpoint in a theory with properties so close to the ones of QCD, where this is of prime importance for future heavy-ion experiments.

To summarize, we have determined for the first time, but still at an exploratory level, the phase diagram of $\mathrm{G}_{2}$ QCD, a non-Abelian gauge theory with fermionic baryons. This opens new avenues for high density studies of the strong force from first-principles calculations. In particular, it will be possible to assess the role of the Fermi statistics at finite baryon density which is of great importance, e.g. for compact stellar objects. One might ask at which relative densities quark or hadron equations of state are more favorable, and thus whether only neutron stars or also quark stars can exist. Given the inflow of new astrophysical observational data on compact stellar objects, including major new discoveries, any insight on a fundamental level would be timely and important. Of course, $\mathrm{G}_{2}$ QCD is not QCD, and such insight can only be qualitative.

It also has to be assessed carefully to what extent the particle spectra of the two differ and what effects this has. However, these ab-initio results provide a novel approach to test investigations of the true QCD phase diagram by effective models and functional methods. If $\mathrm{G}_{2} \mathrm{QCD}$ is continuously deformable to QCD [20, 21, 26], this may proceed along the lines developed for Yang-Mills theory 5 7]: Systematically check approximations and model assumptions in functional methods along this deformation from lattice calculations as long as possible, so that the latter can then be used reliably in the final step towards QCD when the sign problem strikes back.

\section{Acknowledgments}

We are grateful to Simon Hands and Uwe-Jens Wiese 


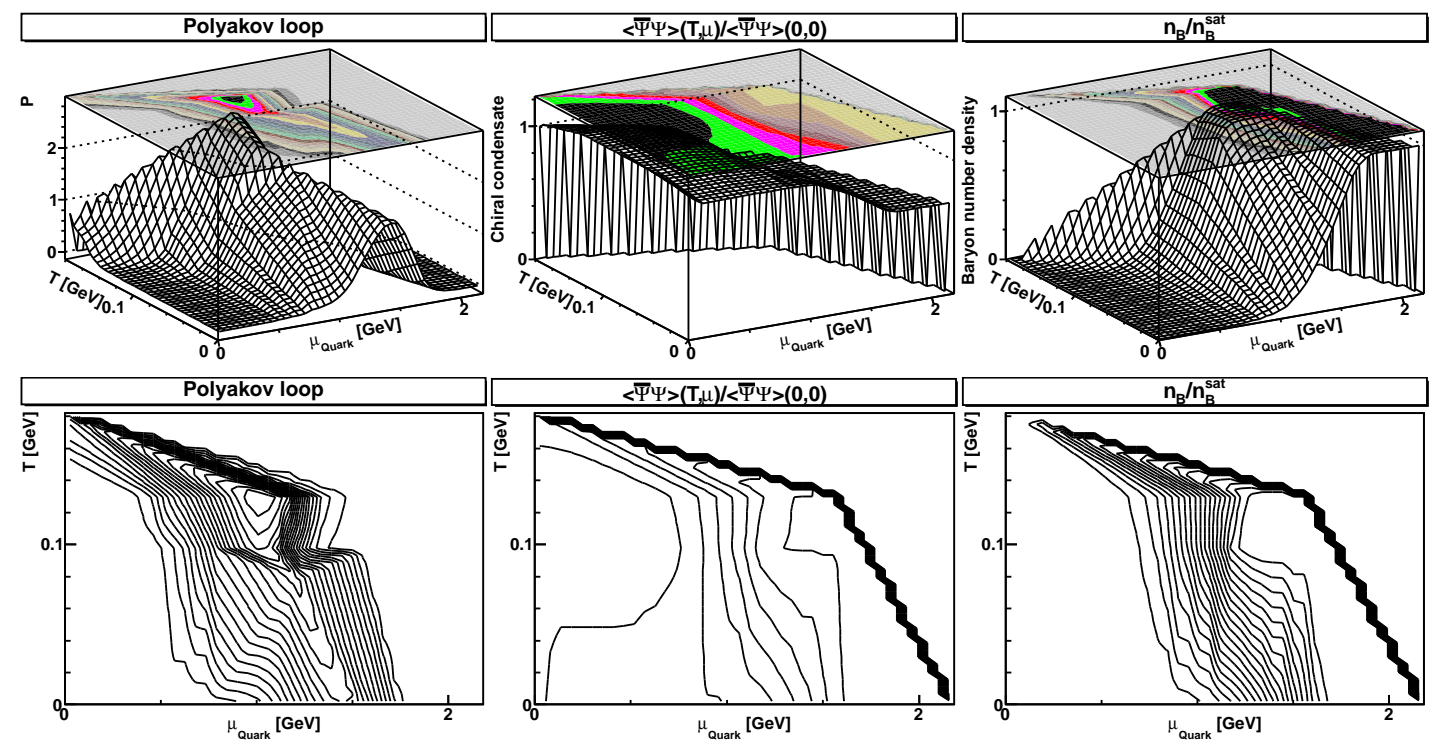

FIG. 5: Interpolated phase diagram for Polyakov loop (left), chiral condensate (middle), and baryon density (right).

for helpful discussions and comments. B.W. was supported by the DFG graduate school 1523-1, A.M. under DFG grant number MA 3935/5-1, A.W. under DFG grant number Wi $777 / 11$, and L.v.S. by the Helmholtz International Center for FAIR within the LOEWE pro- gram of the State of Hesse, the Helmholtz Association Grant VH-NG-332, and the European Commission, FP7PEOPLE-2009-RG No. 249203. Simulations were performed on the LOEWE-CSC at the University of Frankfurt and on the HPC cluster at the University of Jena.
[1] C. Gattringer and C. B. Lang, Quantum chromodynamics on the lattice (Lect. Notes Phys., 2010).

[2] P. de Forcrand, PoS LAT2009, 010 (2009).

[3] S. Leupold et al., Lect. Notes Phys. 814, 39 (2011).

[4] M. Buballa, Phys. Rept. 407, 205 (2005).

[5] J. M. Pawlowski, AIP Conf. Proc. 1343, 75 (2010).

[6] J. Braun, J. Phys. G G39, 033001 (2012).

[7] A. Maas, Phys. Rept. in press (2012), arXiv:1106.3942

[8] C. Bonati, P. de Forcrand, M. D'Elia, O. Philipsen, and F. Sanfilippo, preprint (2012), arXiv:1201.2769

[9] P. de Forcrand and O. Philipsen, Phys. Rev. Lett. 105, 152001 (2010).

[10] P. Cea, L. Cosmai, M. D'Elia, A. Papa, and F. Sanfilippo, preprint (2012), arXiv:1202.5700.

[11] J. Kogut and D. Sinclair, Phys. Rev. D70, 094501 (2004).

[12] P. de Forcrand, M. A. Stephanov, and U. Wenger, PoS LAT2007, 237 (2007).

[13] J. Kogut, M. A. Stephanov, D. Toublan, J. Verbaarschot, and A. Zhitnitsky, Nucl. Phys. B582, 477 (2000).

[14] S. Hands et al., Eur. Phys. J. C17, 285 (2000).

[15] S. Hands, S. Kim, and J.-I. Skullerud, Eur. Phys. J. C48, 193 (2006).

[16] S. Hands, P. Kenny, S. Kim, and J.-I. Skullerud, Eur. Phys. J. A47, 60 (2011).

[17] N. Strodthoff, B.-J. Schaefer, and L. von Smekal, Phys. Rev. D85, 074007 (2012).

[18] P. Braun-Munzinger and J. Wambach, Rev. Mod. Phys. 81, 1031 (2009).

[19] P. de Forcrand and M. Fromm, Phys. Rev. Lett. 104, 112005 (2010).
[20] K. Holland, P. Minkowski, M. Pepe, and U. J. Wiese, Nucl. Phys. B668, 207 (2003).

[21] A. Maas, L. von Smekal, B. Wellegehausen, and A. Wipf, in preparation, 2012.

[22] L. Liptak and Š. Olejník, Phys. Rev. D78, 074501 (2008).

[23] J. Greensite, K. Langfeld, Š. Olejník, H. Reinhardt, and T. Tok, Phys. Rev. D75, 034501 (2007).

[24] M. Pepe and U. J. Wiese, Nucl. Phys. B768, 21 (2007).

[25] B. H. Wellegehausen, A. Wipf, and C. Wozar, Phys. Rev. D83, 016001 (2011).

[26] B. H. Wellegehausen, A. Wipf, and C. Wozar, Phys. Rev. D83, 114502 (2011).

[27] B. H. Wellegehausen, PoS LATTICE 2011, 266 (2011).

[28] G. Cossu, M. D'Elia, A. Di Giacomo, B. Lucini, and C. Pica, JHEP 10, 100 (2007).

[29] J. Danzer, C. Gattringer, and A. Maas, JHEP 01, 024 (2009).

[30] F. Karsch and M. Lutgemeier, Nucl.Phys. B550, 449 (1999).

[31] J. Engels, S. Holtmann, and T. Schulze, Nucl. Phys. B724, 357 (2005).

[32] E. Bilgici, C. Gattringer, E.-M. Ilgenfritz, and A. Maas, JHEP 0911, 035 (2009).

[33] S. Hands, T. J. Hollowood, and J. C. Myers, JHEP 1012, 057 (2010).

[34] L. von Smekal, Nucl. Phys. B (Proc. Suppl.) 228, 179 (2012).

[35] T. D. . Cohen, Phys. Rev. Lett. 91, 222001 (2003).

[36] A. Maas and B. H. Wellegehausen, preprint (2012), arXiv:1210.7950 